\title{
Thorax Computed Tomography Involvement Can Be Predicted by Evaluating the Laboratory Parameters of Patients Admitted to the Emergency Department During the COVID-19 Pandemic Period
}

\author{
(D) Hasan Basri Çetinkaya¹, (1) Hikmet Çoban² \\ 1Department of Emergency Medicine, Balıkesir University, Balıkesir, Turkey \\ ${ }^{2}$ Department of Chest Diseases, Balıkesir University, Balıkesir, Turkey
}

\begin{abstract}
Aim: In this study, we evaluated the predictability of lung parenchymal involvement on computed tomography (CT) with laboratory parameters in patients with confirmed coronavirus disease-2019 (COVID-19) with and without lung parenchymal involvement at the first admission to the emergency department.

Materials and Methods: The study included one hundred and nine patients diagnosed with COVID-19 in the emergency COVID-19 department between April and September 2020. Laboratory parameters and thorax CT images were evaluated to evaluate the severity of the disease in all patients. The relationship between laboratory parameters was analysed in the patient groups with and without CT involvement.

Results: CT involvement was detected in 58 of 109 patients included in the study. There was a significant difference in lymphocyte, monocyte, eosinophil, ferritin, fibrinogen, C-reactive protein (CRP), erythrocyte sedimentation rate (ESR), platelet (PLT) and urea values in the group with $\mathrm{CT}$ involvement. Receiver operator characteristics analysis was performed to evaluate the diagnostic performance of laboratory parameters in CT involvement. Significant diagnostic predictability values were determined for age, lymphocyte, monocyte, eosinophil, ferritin, fibrinogen, CRP, ESR, PLT and urea. The highest area under the curve values was obtained in CRP, ESR and eosinophil parameters.

Discussion: Lymphocyte, monocyte, eosinophil, ferritin, fibrinogen, CRP, ESR, PLT and urea parameters can be used to predict lung involvement in the emergency department in patients with COVID-19 disease. According to these values, thorax CT can be decided for the patients. CRP, ESR and eosinophil parameters provided the highest specificity and sensitivity values in predicting lung involvement.
\end{abstract}

Keywords: COVID-19, lung, tomography, laboratory parameters

\section{Introduction}

Due to the pandemic caused by coronavirus disease-2019 (COVID-19), worldwide cases are increasing day by day and the disease is becoming a global outbreak. As the number of patients increases, it poses great challenges for the health system $(1,2)$. Early diagnosis and treatment continue to be key elements of COVID-19 management. Laboratory and radiological findings of patients diagnosed with COVID-19 are critical in the diagnosis and treatment of the disease (3). A significant correlation has been shown between pulmonary inflammation and lymphocyte, monocyte, CRP, procalcitonin (PCT) values. Thorax computed tomography $(\mathrm{CT})$ is often performed performed to rule out pneumonia in patients who were diagnosed with COVID-19 at emergency service admission. It was found that there was a significant correlation between pulmonary involvement and laboratory results and computed tomography played an important role in the diagnosis, and evaluation of the disease (4). CT scan increases the cost and poses a risk of exposure to medical radiation. In addition to these disadvantages, in hospital emergency services where trauma, and stroke admissions are intense, a large number of patients have to undergo a CT scan in

Cite this article as: Çetinkaya HB, Coban H. Thorax Computed Tomography Involvement Can Be Predicted by Evaluating the Laboratory Parameters of Patients Admitted to the Emergency Department During the COVID-19 Pandemic Period. Eurasian J Emerg Med. 2021;20(2):277-82. 
a limited time. In patients diagnosed or suspected of COVID-19, 30 minutes or more is required for post-CT cleaning. This long period may cause disruptions in CT scans in routine emergency practice. This cleaning time delays access to CT for other patients in the emergency department. Depending on the prolongation of this period, other patient groups will be more likely to be exposed to COVID-19 $(5,6)$.

In this study, it was planned to determine the predictability of COVID-19 lung involvement with routine laboratory evaluations in the emergency department. Thus, by looking at the laboratory tests, it can be estimated which patients may have a higher risk of COVID-19 lung involvement. With this estimation, computed tomography can be performed only in patients who are thought to have lung involvement. Patients who do not require thorax CT will be excluded.

\section{Materials and Methods}

A total of 109 patients with PCR positivity who applied to the emergency department of Balıkesir University Medical Faculty Hospital between April and September 2020 were included in the study. Patients with chronic disease (cirrhosis, cardiac failure, chronic renal failure) that may affect clinical and laboratory parameters and patients using drugs (such as steroids) that may affect laboratory values were excluded from the study. Children and pregnant women were excluded from the study. Laboratory data were obtained from detailed medical records. Complete blood count, kidney and liver function tests, inflammation parameters such as CRP, ESR, PCT, ferritin, D-dimer and fibrinogen were recorded at the first admission to the emergency department. NLR value was calculated. CT examinations were performed with a 16-line multi-detector CT scanner (Siemens Somatom Sensation; Siemens, Erlangen, Germany). CT images of all patients included in the study were evaluated and cases with bacterial pneumonia or pulmonary oedema were excluded from the study. This retrospective chart review study involving human participants was in accordance with the ethical standards of the institutional and national research committee and with the 1964 Helsinki Declaration and its later amendments or comparable ethical standards, and the local ethics committee approved this study (decision number: 2020/182, date: 14.10.2020).

\section{Statistical Analysis}

Shapiro-Wilk test was used to test the normality of variables. Continuous variables were presented as median ( $1^{\text {st }}$ quartile $-3^{\text {rd }}$ quartile) values since the data were not normally distributed. Mann-Whitney's U-test was used for comparison of two independent groups. Categorical variables were expressed with numbers and percentages. Comparisons between the groups were performed with Pearson chi-square test for categorical variables. Risk factors were also evaluated with binary logistic regression analysis. Receiver operating characteristics (ROC) curve analysis was performed to evaluate and compare the performances of diagnostic markers. Significance level was taken as $\alpha=0.05$. Statistical analyses were performed with IBM SPSS Statistics version 22.0 (IBM Corp., Armonk, NY, USA) and MedCalc version 12.3.0.0.

\section{Results}

The study included 109 patients, 58 (53.21\%) of whom had positive chest CT findings and 51 (46.79\%) of whom had negative chest CT findings. Among 109 patients, 45 (41.28\%) were male, 64 $(58.72 \%)$ were female, and the median age was $56\left(1^{\text {st }}\right.$ quartile $3^{\text {rd }}$ quartile: $38-68$ ) years.

There was a significant difference between chest $\mathrm{CT}$ positive and negative groups in terms of age, lymphocytes, monocytes, eosinophils, ferritin, fibrinogen, CRP, ESR, PLT and urea. There was no significant difference between the two groups in terms of NLR and the other variables (Table 1).

We conducted backward conditional logistic regression analysis by including the variables into the model which were found statistically significant in univariate analysis. In the last model remaining variables were eosinophils, monocytes, ferritin, ESR and urea. The last model was statistically significant $(p<0.001$ for Omnibus test; $p=0.917$ for Hosmer \& Lemeshow test). Eosinophils was not statistically significant in the model. One unit decrease in the monocytes increased the risk of chest CT positivity 1.006 times $(p=0.001)$, one unit increase in the ferritin increased the risk of chest $C T$ positivity 1.014 times $(p=0.021)$, one unit increase in the ESR increased the risk of chest CT positivity 1.069 times $(p=0.001)$, and one unit increase in the urea increased the risk of chest CT positivity 1.090 times ( $p=0.013)$, compared to chest CT negative patients (Table 2).

We performed ROC curve analyses to evaluate the diagnostic performances of age, WBC, neutrophil count, lymphocyte count, NLR, monocyte count, eosinophil count, ferritin, PCT, fibrinogen, D-dimer, CRP, ESR, PLT, RDW, urea, creatinine, AST and ALT in discriminating patients with positive and negative CT imaging findings. Optimal cut-off values were obtained according to Youden J index, corresponding sensitivity and specificity values are given. Significant diagnostic performances were obtained for age, lymphocyte count, monocyte count, eosinophil count, ferritin, fibrinogen, CRP, ESR, PLT and urea. Three largest area under the curves (AUCS) were obtained for CRP, ESR and eosinophil count. (Table 3, Figure 1). 


\begin{tabular}{|c|c|c|c|}
\hline Variable & CT positive & CT negative & $p$-value \\
\hline \multicolumn{4}{|l|}{ Gender } \\
\hline Male & $24(41.38)$ & $21(41.18)$ & \multirow[t]{2}{*}{1.000} \\
\hline Female & $34(58.63)$ & $30(58.82)$ & \\
\hline Age (years) & $60(48-69)$ & $44(31-67)$ & 0.016 \\
\hline WBC $(\mu \mathrm{L})$ & $5,350(4,500-6,600)$ & $6,100(4,800-7,600)$ & 0.125 \\
\hline Neutrophils $(\mu \mathrm{L})$ & $3,366(2,711-4,541)$ & $3,692(2,462-4,680)$ & 0.762 \\
\hline Lymphocytes ( $\mu \mathrm{L})$ & $1,244(694-1,611)$ & $1,624(1,044-1,996)$ & 0.006 \\
\hline NLR & $2.87(1.89-4.49)$ & $2.26(1.53-3.95)$ & 0.109 \\
\hline Monocytes $(\mu \mathrm{L})$ & $413(337-555)$ & $490(411-755)$ & 0.004 \\
\hline Eosinophils ( $\mu \mathrm{L})$ & $12(4-36)$ & $61.00(25-112)$ & $<0.001$ \\
\hline Ferritin (ng/mL) & 77 (39-179) & 38 (18-73) & 0.001 \\
\hline $\mathrm{PCT}(\mathrm{ng} / \mathrm{mL})$ & $0.09(0.04-0.13)$ & $0.07(0.04-0.10)$ & 0.430 \\
\hline Fibrinogen (mg/dL) & $399(288-516)$ & $300(260-336)$ & $<0.001$ \\
\hline D-dimer (ng/mL) & $213(142-270)$ & $153(130-234)$ & 0.051 \\
\hline $\mathrm{CRP}(\mathrm{mg} / \mathrm{L})$ & $22(6-48)$ & $3(2-6)$ & $<0.001$ \\
\hline $\operatorname{ESR}(\mathrm{mm} / \mathrm{h})$ & $44(24-67)$ & $22(13-33)$ & $<0.001$ \\
\hline $\operatorname{PLT}\left(10^{3} / \mathrm{mm}^{3}\right)$ & $203(166-246)$ & $227(197-273)$ & 0.013 \\
\hline RDW (\%) & $14.2(13.4-15.2)$ & $14.0(13.2-15.2)$ & 0.447 \\
\hline Urea (mg/dL) & $31(26-40)$ & $24(19-34)$ & 0.004 \\
\hline Creatinine (mg/dL) & $0.94(0.81-1.07)$ & $0.89(0.78-1.02)$ & 0.214 \\
\hline AST (IU/L) & $26(22-33)$ & $24(21-37)$ & 0.309 \\
\hline ALT (IU/L) & $22(15-28)$ & $21(14-38)$ & 0.995 \\
\hline \multicolumn{4}{|c|}{$\begin{array}{l}\text { Data given as median }\left(1^{\text {st }} \mathrm{Q}-3^{\text {rd }} \mathrm{Q}\right) \text { or } \mathrm{n}(\%) \\
\text { CT: Computed tomography, WBC: White blood cell distribution width, NLR: neutrophil lymphocyte ratio, PCT: Procalcitonin, CRP: C-reactive protein, ESR: Erythrocyte } \\
\text { sedımentatıon rate, PLT: Platelet, RDW: Red cell distribution width, AST: Aspartat aminotransferaz, ALT: Alanin aminotransferaz, Q: Quartile, } \mathrm{n} \text { : Number }\end{array}$} \\
\hline
\end{tabular}

\begin{tabular}{|l|l|l|l|l|}
\hline Table 2. Results of logistic regression analysis \\
\hline $\begin{array}{l}\text { Independent } \\
\text { variables }\end{array}$ & $\mathbf{p}$-value & $\mathbf{0 R}$ & $\mathbf{9 5 \%}$ CI for OR \\
\cline { 3 - 5 } & & & Lower & Upper \\
\hline Eosinophils & 0.071 & 1.008 & 0.999 & 1.016 \\
\hline Monocytes & 0.001 & 1.006 & 1.002 & 1.009 \\
\hline Ferritin & 0.021 & 1.014 & 1.002 & 1.025 \\
\hline ESR & 0.001 & 1.069 & 1.027 & 1.113 \\
\hline Urea & 0.013 & 1.090 & 1.018 & 1.168 \\
\hline OR: Odds ratio, CI: Confidence interval, ESR: Erythrocyte sedimentation rate \\
\hline
\end{tabular}

\section{Discussion}

In our study, there was a significant difference in age, lymphocyte count, monocyte count, eosinophil count, platelet count, ferritin, fibrinogen, CRP, ESR, and urea parameters between patients with and without lung involvement in thoracic CT. Sensitivity of COVID-19 pneumonia in CT was found to be $75 \%$ or more in patients with lymphocyte count $\leq 1610(\mu \mathrm{L})$, age $>44$, eosinophil

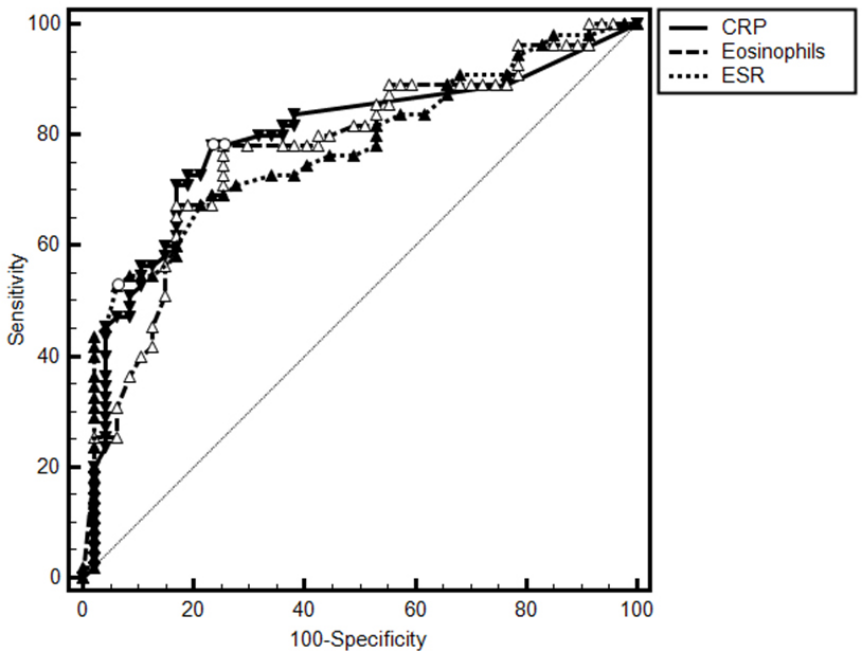

Figure 1. ROC curve for CRP, ESR and eosinophils

ROC: Receiver operating characteristics, CRP: C-reactive protein, ESR: Erythrocyte sedimentation rate 
Table 3. ROC curve analysis results for thorax CT results

\begin{tabular}{|c|c|c|c|c|c|c|}
\hline & AUC & p-value & Cut-off value & $\begin{array}{l}\text { Youden J } \\
\text { index }\end{array}$ & $\begin{array}{l}\text { Sensitivity } \\
(95 \% \mathrm{CI})\end{array}$ & $\begin{array}{l}\text { Specificity } \\
(95 \% \mathrm{CI})\end{array}$ \\
\hline Age (years) & 0.634 & 0.015 & $>44$ & 0.305 & 77.59 (64.7-87.5) & $52.94(38.5-67.1)$ \\
\hline Lymphocytes ( $\mu \mathrm{L})$ & 0.654 & 0.004 & $\leq 1610$ & 0.274 & $75.44(62.2-85.9)$ & $52.00(37.4-66.3)$ \\
\hline Monocytes $(\mu \mathrm{L})$ & 0.664 & 0.002 & $\leq 466$ & 0.289 & $64.91(51.1-77.1)$ & $64.00(49.2-77.1)$ \\
\hline Eosinophils ( $\mu \mathrm{L})$ & 0.750 & $<0.001$ & $\leq 37$ & 0.472 & 77.19 (64.2-87.3) & $70.00(55.4-82.1)$ \\
\hline Fibrinogen (mg/dL) & 0.711 & $<0.001$ & $>383$ & 0.452 & $53.57(39.7-67.0)$ & $91.67(80.0-97.7)$ \\
\hline CRP (mg/L) & 0.783 & $<0.001$ & $>6.26$ & 0.523 & $75.86(62.8-86.1)$ & $76.47(62.5-87.2)$ \\
\hline $\operatorname{ESR}(\mathrm{mm} / \mathrm{h})$ & 0.762 & $<0.001$ & $>43$ & 0.452 & $53.57(39.7-67.0)$ & $91.67(80.0-97.7)$ \\
\hline $\operatorname{PLT}\left(10^{3} / \mathrm{mm}^{3}\right)$ & 0.638 & $<0.001$ & $\leq 170$ & 0.254 & $29.31(18.1-42.7)$ & 96.08 (86.5-99.5) \\
\hline
\end{tabular}

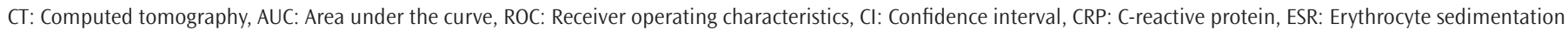
rate, PLT: Platelet

count $\leq 37(\mu \mathrm{L}), \mathrm{CRP}>6.26(\mathrm{mg} / \mathrm{L})$, and urea $>26(\mathrm{mg} / \mathrm{dL})$. In $\mathrm{CT}$, the specificity of COVID-19 pneumonia was found to be $90 \%$ or more in patients with fibrinogen $>383(\mathrm{mg} / \mathrm{dL})$, sedim $>43(\mathrm{~mm} / \mathrm{h})$ and platelet $\leq 170\left(10^{3} / \mathrm{mm}^{3}\right)$. In the retrospective conditional logistic regression analysis, eosinophil count, monocyte count, ferritin, ESR and urea parameters were determined in the last model by including the variables found to be statistically significant in the univariate analysis. Although eosinophil count was not statistically significant in the model, a one-unit decrease in monocyte count value significantly increased the risk of thoracic CT positivity by 1.006 times, a one-unit increase in ferritin increased the risk of thoracic CT positivity by 1.014 times, a oneunit increase in ESH increased the risk of thoracic CT positivity by 1.069 times and a one-unit increase in the urea value increased the risk of thoracic CT positivity by 1.090 times.

Lymphopenia is a laboratory finding of COVID-19 infection and was detected in $63 \%$ of cases (7). It has been suggested that the degree of lymphopenia may predict the severity, progression and prognosis of COVID-19 (8,9). In our study, a significant difference was found between lymphocyte count between patients with and without involvement in thoracic CT. In patients with lymphocyte count $\leq 1610(\mu \mathrm{L})$, the sensitivity and specificity was found $75.44 \%$, and $52 \%$, respectively in predicting involvement in thoracic CT.

Previous studies have shown that two-thirds of patients infected with COVID-19 have elevated CRP. In the early stage of the disease, CRP was correlated with lung lesions, severity of pneumonia, and overall disease severity (9-13). In our study, a significant difference was found between CRP values between patients with and without involvement in thoracic $\mathrm{CT}$. Predicting of involvement of thoracic CT in patients with CRP $>6.26(\mathrm{mg} / \mathrm{L})$ sensitivity and specificity were $75.86 \%$, and $76.47 \%$, respectively.

A meta-analysis evaluating severe and non-severe confirmed COVID-19 cases showed significant reductions in monocyte, eosinophil and platelet levels in patients with severe disease (13). In our study, a significant difference was found between monocyte, eosinophil and platelet values between patients with and without involvement in thoracic CT. The sensitivity and specificity in predicting thoracic $\mathrm{CT}$ involvement were $64.91 \%$ and $64 \%$, respectively, in patients with monocytes $\leq 466(\mu \mathrm{L})$, and a one-unit decrease in monocytes increased the risk of thoracic CT positivity 1.006 times. The sensitivity and specificity in predicting thoracic CT involvement were $77.19 \%$ and $70 \%$, respectively, in patients with eosinophils $\leq 37(\mu \mathrm{L})$. The sensitivity and specificity in predicting thoracic CT involvement were $29.31 \%$ and $96.08 \%$, respectively, in patients with platelets $\leq 170\left(10^{3} / \mathrm{mm}^{3}\right)$.

Ferritin levels have been shown to increase significantly compared to those without severe COVID-19 disease and patients with mortality have higher ferritin levels $(9,12,14)$. In our study, a significant difference was found between ferritin values between patients with and without involvement in thoracic CT. The sensitivity and specificity in predicting thoracic CT involvement were $61.40 \%$ and $75 \%$, respectively, in patients with ferritin $>65$ $(\mathrm{ng} / \mathrm{mL})$, and a one-unit increase in ferritin value showed that it increased the risk of thoracic CT positivity 1.014 times.

Fibrinogen levels have been shown to be higher in COVID-19 patients compared to healthy controls, as well as higher in critical COVID-19 patients compared to mild or moderate cases $(12,13,15)$. However, fibrinogen level may not have a predictive value for mortality in COVID-19 patients (16). It is recommended that fibrinogen be evaluated together with D-dimer levels to 
have more appropriate prognostic assumptions (17). A significant correlation has been reported between the severity of COVID-19 disease and D-dimer serum level $(9,12,13,18,19)$. However, in a cohort, it was shown that there was no difference between the severity of the disease and D-dimer level in COVID-19 patients (20). D-dimer has been emphasized to have a promising value for guiding anticoagulation strategies in the treatment of COVID-19 (17). In our study, a significant difference was found in fibrinogen values between patients with and without involvement in thoracic CT. In patients with a fibrinogen value $>383 \mathrm{mg} / \mathrm{dL}$, sensitivity and specificity was $53.57 \%$ and $91.67 \%$, respectively, in predicting thorax CT involvement. In our study, D-Dimer levels were found to be similar between patients with and without involvement in thoracic $\mathrm{CT}$. This may be due to the small number of severe patients in our study.

Among the laboratory tests used for the evaluation of the acute phase reaction reflecting the inflammatory condition, ESR is considered the least specific. It is known to be affected by a large number of other physiological and pathophysiological conditions and its use is limited to a few specific clinical conditions (21). Nevertheless, ESR is still persistently used in routine laboratory patient examinations regardless of the clinical problem (22). It has been shown that there is a significant difference in ESR values between severe and non-severe COVID-19 cases $(9,13,23)$. In our study, a significant difference was found between ESR value among patients with and without involvement in thoracic CT. The sensitivity and specificity in predicting thoracic CT involvement were $53.57 \%$ and $91.67 \%$, respectively, in patients with ESR $>43 \mathrm{~mm} / \mathrm{h}$, and a one-unit increase in ESR increased the risk of thoracic CT positivity by 1.069 times.

When severe COVID-19 cases and mild cases were compared, statistically significantly higher AST, ALT, creatinine, and urea levels were found $(9,13)$. In our study, a significant difference was found between blood urea values among patients with and without involvement in thoracic $\mathrm{CT}$, and the sensitivity and specificity in predicting thoracic CT involvement were $74.14 \%$ and $60.78 \%$, respectively, in patients with urea values $>26 \mathrm{mg} / \mathrm{dL}$. A one-unit increase in urea value increased the risk of thoracic CT positivity 1.090 times. In our study, creatinine, AST and ALT levels were found to be similar between patients with and without involvement in thoracic CT.

RDW has been shown to be a prognostic predictor for severe COVID-19 patients (24). When severe COVID-19 cases and mild cases were compared, it was reported that PCT value was higher and was a poor prognostic marker $(9,12,13)$. The meta-analysis evaluating severe and non-severe confirmed COVID-19 cases showed increased neutrophil and NLR rates and no difference was found between leukocyte values (13). In our study, no significant relationship was found between leukocyte, neutrophil, NLR and RDW values between patients with and without pulmonary involvement in thoracic CT.

\section{Study Limitations}

Our study has its limitations; firstly, the number of patients included in the study was small. Secondly, this study was a single center study, and it is not capable of evaluating various ethnic differences, thus preventing the generalized use of the study results.

\section{Conclusion}

As a conclusion, in the first evaluation in the emergency department, a significant difference was found in lymphocytes, monocytes, eosinophil, ferritin, fibrinogen, CRP, ESR, PLT and urea values between patients with and without pulmonary involvement in thoracic CT, and eosinophil count, ESR and CRP values provided the highest AUC values in predicting thoracic CT involvement. It can be predicted that patients with ESR $>43$ $\mathrm{mm} / \mathrm{h}$ and CRP $>6.26 \mathrm{mg} / \mathrm{L}$ and eosinophil count $\leq 37 \mu \mathrm{L}$ will have a high probability of lung involvement. The need for thoracic CT during the pandemic can be planned according to these criteria.

\section{Ackowledgements}

The authors would like to thank Associate Professor Deniz Sığırlı of Bursa Uludağ University School of Medicine Biostatistics Department for her invaluable help in doing statistical analyses.

\section{Ethics}

Ethics Committee Approval: Balıkesir University Clinical Studies Ethics Committee approved this study (decision number: 2020/182, date: 14.10.2020).

Informed Consent: Not applicable since the study is a retrospective chart review study.

Peer-review: Externally peer-reviewed.

\section{Authorship Contributions}

Surgical and Medical Practices: H.B.C.., H.C.., Concept: H.B.C.., H.C.., Design: H.B.Ç., H.Ç., Data Collection and/or Processing: H.B.C.., H.C.., Analysis and/or Interpretation: H.B.C.., H.C.., Literature Search: H.B.C., H.Ç., Writing: H.B.C,., H.Ç.

Conflict of Interest: No conflict of interest was declared by the authors.

Financial Disclosure: The authors declared that this study received no financial support. 


\section{References}

1. Huang C, Wang Y, Li X, Ren L, Zhao J, Hu Y, et al. Clinical features of patients infected with 2019 novel coronavirus in Wuhan, China. Lancet. 2020;395:497506.

2. Yaqian M, Lin W, Wen J, Chen G. Clinical and pathological characteristics of 2019 novel coronavirus disease (COVID-19): A systematic review. 2020 medRxiv. doi: 10.1101/2020.02.20.20025601

3. Ghayda RA, Lee J, Lee JY, Kim DK, Lee KH, Hong SH, et al. Correlations of clinical and laboratory characteristics of COVID-19: a systematic review and meta-analysis. Int J Environ Res Public Health. 2020;17:5026.

4. Wu J, Wu X, Zeng W, D Guo, Z Fang, L Chen, et al. Chest CT findings in patients with coronavirus disease 2019 and its relationship with clinical features. Invest Radiol. 2020;55:257-61.

5. Al-Tawfiq JA, Memish ZA. Diagnosis of SARS-CoV-2 Infection based on CT scan vs. RT-PCR: reflecting on experience from MERS-CoV. J Hosp Infect. 2020;105:154-55.

6. Majidi H, Niksolat F. Chest CT in patients suspected of COVID-19 infection: a reliable alternative for RT-PCR. Am J Emerg Med. 2020;38:2730-32.

7. Qin C, Zhou L, Hu Z, Zhang S, Yang S, Tao Y, et al. Dysregulation of immune response in patients with COVID-19 in Wuhan, China. Clin. Infect. Dis. 2020;71:762-68.

8. Tan L, Wang Q, Zhang D, Ding J, Huanget Q, Tang YQ, et al. Lymphopenia predicts disease severity of COVID-19: A descriptive and predictive study. Signal. Transduct. Target Ther. 2020;5:33.

9. Mudatsir M, Fajar JK, Wulandari L, Soegiarto G, Ilmawan M, Purnamasari Y, et al. Predictors of COVID-19 severity: a systematic review and meta-analysis. 2020;9:1107.

10. Wang L. C-reactive protein levels in the early stage of COVID-19. Med Mal Infect. 2020:50:332-4.

11. Shi $\mathrm{H}$, Han X, Jiang N, Cao Y, Alwalid O, Gu J, et al. Radiological findings from 81 patients with COVID-19 pneumonia in Wuhan, China: A descriptive study. Lancet Infect Dis. 2020;20:425-34.

12. Huang I, Pranata R, Lim MA, Oehadian A, Alisjahbana B. C-reactive protein, procalcitonin, D-dimer, and ferritin in severe coronavirus disease-2019: a meta-analysis. Ther Adv Respir Dis. 2020;14:1753466620937175.
13. Ghahramani S, Tabrizi R, Lankarani KB, Kashani SMA, Rezaei S, Zeidi N, et al. Laboratory features of severe vs. non-severe COVID-19 patients in Asian populations: a systematic review and meta-analysis. Eur J Med Res. 2020;25:30. doi: 10.1186/s40001-020-00432-3

14. Cheng L, Li H, Li L, Liu C, Yan S, Chen H, et al. Ferritin in the coronavirus disease 2019 (COVID-19): A systematic review and meta-analysis. J Clin Lab Anal. 2020;34:e23618.

15. Han H, Yang L, Liu R, Liu F, Wu KL, Li J, et al. Prominent changes in blood coagulation of patients with SARS-CoV-2 infection. Clin Chem Lab Med. 2020;58:1116-20.

16. Tang N, Li D, Wang X, Sun Z. Abnormal coagulation parameters are associated with poor prognosis in patients with novel coronavirus pneumonia. J Thromb Haemost. 2020;18:844-47.

17. Hayıroğlu Mi, Çınar T, Tekkeșin Ai. Fibrinogen and D-dimer variances and anticoagulation recommendations in Covid-19: current literature review. Rev Assoc Med Bras. 2020;66:842-48.

18. Chen G, Wu D, Guo W, Cao Y, Huang D, Wang H, et al. Clinical and immunological features of severe and moderate coronavirus disease 2019. J Clin Invest. 2020;130:2620-29.

19. Zhang JJ, Dong X, Cao YY, Yuan YD, Yang YB, Yan YQ, et al. Clinical characteristics of 140 patients infected with SARS-CoV-2 in Wuhan, China. Allergy. 2020;75:1730-41

20. Zhou Y, Zhang Z, Tian J, Xiong S. Risk factors associated with disease progression in a cohort of patients infected with the 2019 novel coronavirus. Ann Palliat Med. 2020;9:428-36.

21. Lapić I, Padoan A, Bozzato D, Plebani M. Erythrocyte sedimentation rate and C-reactive protein in acute inflammation: meta-analysis of diagnostic accuracy studies. Am J Clin Pathol. 2020;153:14-29.

22. Horton S, Fleming KA, Kuti M, Looi LM, Pai SA, Sayed S, et al. The top 25 laboratory tests by volume and revenue in five different countries. Am J Clin Pathol. 2019;151:446-51.

23. Lapić I, Rogić $\mathrm{D}$, Plebani M. Erythrocyte sedimentation rate is associated with severe coronavirus disease 2019 (COVID-19): a pooled analysis. Clin Chem Lab Med. 2020;58:1146-48.

24. Wang C, Zhang H, Cao X, Deng R, Ye Y, Fu Z, et al. Red cell distribution width (RDW): a prognostic indicator of severe COVID-19. Ann Transl Med. 2020;8:1230. doi: 10.21037/atm-20-6090 